\title{
INDEX TO VOLUME 73
}

\section{RESEARCH ANNOUNCEMENTS}

Akemann, C. A. Projections onto separable $C^{*}$-subalgebras of a $W^{*}$-algebra, 925.

Almgren, F. J., Jr. Existence and regularity of solutions to elliptic calculus of variations problems among surfaces of varying topological type and singularity structure, 576.

Ar, Ergun. The solution spaces for certain equations of the scattering theory, 663.

Armstrong, M. A. and Zeeman, E. C. Piecewise linear transversality, 184.

Aronson, D. G. Bounds for the fundamental solution of a parabolic equation, 890.

Arveson, William B. An algebraic conjugacy invariant for measure preserving transformations, 121.

Asplund, Edgar. Positivity of duality mappings, 200.

See Namioka, I.

Assmus, E. F. Jr. and Mattson, H. F., Jr. Erratum to Disjoint Steiner systems associated with the Mathieu groups, 496.

Auslander, Louis and Baumslag, Gilbert. Automorphism groups of finitely generated nilpotent groups, 716.

and Kostant, B. Quantization and representations of solvable Lie groups, 692.

Babbitt, Donald G. Wiener integral representations for certain semigroups which have infinitesimal generators with matrix coefficients, 394.

Baum, Leonard E. and Eagon, J. A. An inequality with applications to statistical estimation for probabilistic functions of Markov processes and to a model for ecology, 360 .

Baumslag, Gilbert. Residually finite one-relator groups, 618.

- Some groups that are just about free, 621. See Auslander, Louis.

Berger, Melvyn S. An application of the calculus of variations in the large to the equations of nonlinear elasticity, 520.

- On nonlinear perturbations of the eigenvalues of a compact self-adjoint operator, $704 ; 987$.

Boardman, J. M. On manifolds with involution, 136.

Braden, Bart. Restricted representations of classical Lie algebras of types $A_{2}$ and $B_{2}, 482$.

Bragg, L. R. The radial heat equation with pole type data, 133.

Bredon, Glen E. Equivariant cohomology theories, 266.

Equivariant stable stems, 269.

Browder, Felix E. Existence and perturbation theorems for nonlinear maximal monotone operators in Banach spaces, 322.

Nonlinear accretive operators in Banach spaces, 470.

Nonlinear equations of evolution and nonlinear accretive operators in Banach spaces, 867.

Nonlinear mappings of nonexpansive and accretive type in Banach spaces, 875.

Browder, W. and Livesay, G. R. Fixed point free involutions on homotopy spheres, 242.

Bryant, Jack. On convolution and Fourier series, 149.

Chandrasekharan, K. and Narasimhan, Raghavan. On lattice-points in a random sphere, 68.

Chavel, Issac. On normal Riemannian homogeneous spaces of rank 1, 477.

Chen, Kou-Tsai. Algebraization of ilerated integration along paths, 975.

Iterated path integrals and generalized paths, 935. 
Chorin, A. J. The numerical solution of the Navier-Stokes equations for an incompressible fluid, 928.

Chrislock, J. L. and Tamura, T. Subdirect products of semigroups and rectangular bands, 637.

Chuckrow, Vicki. Schottky groups and limits of Kleinian groups, 139.

Chui, Charles Kam-Tai. Bounded approximation by polynomials with restricted zeros, 967.

Claborn, Luther and Fossum, Robert. Higher rank class groups, 233.

Coburn, L. A. The $C^{*}$-algebra generated by an isometry, 722.

Crimmins, T. and Rosenthal, P. On the decomposition of invariant subspaces, 97.

DeFigueiredo, D. G. and Karlovitz, L. A. On the radial projection in normed spaces, 364.

DeGroot, J. An isomorphism principle in general topology, 465.

DeWilde, M. See Garnir, H. G.

Diaz, J. B. and Metcalf, F. T. On the structure of the set of subsequential limit points of successive approximations, 516.

Douglas, R. G., Shapiro, H. S. and Shields, A. L. On cyclic vectors of the backward shift, 156.

Dubois, D. W. Note on Artin's solution of Hilbert's 17th problem, 540.

Eagon, J. A. See Baum, Leonard E.

Earle, Clifford, J. The contractibility of certain Teichmulller spaces, 434.

and Eells, J. The diffeomorphism group of a compact Riemann surface, 557.

Eells, J. See Earle, Clifford, J.

Effros, E. G. and Hahn, Frank. Locally compact transformation groups and $C^{*}$-algebras, 222.

Faith, Carl. A general Wedderburn theorem, 65.

Faris, W. G. The Trotter product formula for perturbations of semibounded operators, 211.

Farkas, H. M. Automorphisms of compact Riemann surfaces and the vanishing of theta constants, 231.

Farrell, F. T. The obstruction to fibering a manifold over a circle, 737.

and Hsiang W. C. H-cobordant manifolds are not necessarily homeomorphic, 741.

Faulkner, J. R. The inner derivations of a Jordan algebra, 208.

Ferrar, J. C. On Lie algebras of type $E_{6}, 151$.

Fisher, Michael. Singular integrals on Hilbert space, 428; 987.

Forster, Otto. Some remarks on parallelizable Stein manifolds, 712.

Fossum, Robert. See Claborn, Luther.

Freeman, R. S. Erratrum to On the spectrum and resolvent of homogeneous elliptic differential operators with constant coefficients, 496.

Freyd, Peter. The Grothendieck groups for stable homotopy is free, 84 .

Friedman, Marcelle. Quasi-periodic solutions of nonlinear ordinary differential equations with small damping, 460.

Frolik, Zdenck. Sums of ultrafilters, 87.

Furstenberg, Harry. Poisson boundaries and envelopes of discrete groups, 350.

Galvin, Fred. Reduced products, Horn sentences and decision problems, 59.

Garnir, H. G., DeWilde, M. and Schmets, J. Constructive proof of the existence of multiplicative functionals in commutative separable Banach algebras, 564.

Gerstenhaber, Murray. The third cohomology group of a ring and the commutative cohomology theory, 950.

Giffen, C. H. On transformations of the 3-sphere fixing a knot, 913. 
Gilmore, Maurice E. Complex Stiefel manifolds, some homotopy groups and vector fields, 630.

Gitler, S. and Mahowald, M. The immerison of manifolds, 696.

Glimm, J. and Lax. P. D. Decay of solutions of systems of hyperbolic conservation laws, 105.

Hermann, Robert. Differential equations of the matrix element of the degenerate series, 899.

Herrera, M. E. de Rham theorems on semianalytic sets, 414 .

Hahn, Frank. See Effros, E. G.

Hahn, K. T. and Mitchell, Josephine. Generalization of Schrwarz-Pick lemma to invariant volume in a Kahler manifold, 668.

Halpern, Benjamin. Fixed points of nonexpanding maps, 957.

Hannula, T. A. Ralley, T. G. and Reiner, I. Modular representation algebras, 100.

Hanson, D. L. A relation between moment generating functions and convergence rates in the law of large numbers, 95.

Harrold, O. G. Jr. and Seebeck, C. L. Jr. Locally weakly flat spaces, 457.

Hodgson, J. P. E. Automorphisms of thickenings, 678.

Hörmander, Lars. Generators for some rings of analytic functions, 943.

Hsiang, W. C. see Garrell, F. T.

Hsiang, Wu-yi. The natural metric on $S O(n) / S O(n-2)$ is the most symmetric metric, 55.

Johnson, B. E. A comnutative semisimple annihilator Banach algebra which is not dual, 407.

The uniqueness of the (complete) norm topology, 537.

Johnson, J. L. Global continuous solutions of hyperbolic systems of quasi-linear equations, 639.

- and Smoller, J. A. Global solutions of certain hyperbolic systems of quasi-linear equations, 666.

Kahn, Donald W. Imbedding the stable homotopy category, 649.

Chern numbers and oriented homotopy types, 932.

Karlovitz, L. A. See De Figueiredo, D. G.

Kato, Tosio, Demicontinuity, hemicontinuity and monotonicity. II, 886.

Similarity for sequences of projections, 904.

Kaufman, Robert. An example in the calculus of Fourier transforms, 357.

Kelleher, J. and Taylor, B. A. An application of the Corona Theorem to some rings of entire functions, 246.

Kemeny, J. G. and Snell, J. L. Excessive functions of continuous time Markov chains, 216.

Kendall, David G. Delphic semigroups, 120.

Kessler, Irving, Semidempotent measures on abelian groups, 258.

Khabbaz, Samir A. and Pitcher, Everett. Critical submanifolds of differentiable mappings, 164.

Critical submanifolds of differentiable mappings. II, 310.

Kobayashi, Shoshichi. Intrinsic metrics on complex manifolds, 347.

Koppelman, Walter. The Cauchy integral for differential forms, 554.

The Cauchy integral for functions of several complex variables, 373.

Kostant, B. See Auslander, Louis.

Kripke, Saul. See Pour-El, Marian Boykan,

Lallement, Gerard and Petrich, Mario. Structure of a class of regular semigroups and rings, 419.

Lamperti, John. Continuous state branching processes, 382.

Lax, P. D. See Glimm, J. 
Leadbetter, M. R. A note on the moments of the number of axis-crossings by a stochastic process, 129.

Lipsman, Ronald L. Uniformly bounded representations of SL $(2, C), 652$.

Littman, Walter, $A$ connection between $\alpha$-capacity and m-p polarity, 862.

Livesay, G. R. See Browder, W.

Loos, Ottmar, Reflexion spaces and homogeneous symmetric spaces, 250.

Lopez, William. An example in the fixed point theory of polyhedra, 922.

Lopez de Medrano, Santiago. Involutions of homotopy spheres and homology 3-spheres, 727.

Loscalzo, Frank R. and Talbot, Thomas D. Spline function approximations for solutions of ordinary differential equations, 438.

Luxemburg, W. A. J. Is every integral normal? 685.

Mahowald, M. and R. James Milgram, Embedding projective spaces, 644. See S. Gitler.

Mandrekar, V. and Nadkarni, M. Quasi-invariance of analytic measures on compact groups, 915.

Manis, Merle S. Extension of valuation theory, 735.

Marcus, Marvin, $A$ theorem on rank with applications to mappings on symmetry classes of tensors, 675.

Margulies, William. Analytic singular integral operators, 391.

Mattson, H. F. Jr. See Assmus, F. J.

Max, Nelson L. Homeomorphisms of $S^{n} \times S^{1}, 939$.

McLaughlin, T. G. Hereditarily retraceable isols, 113.

McMillan, D. R. Jr. Strong homotopy equivalence of 3-manifolds, 718.

Melzak, Z. A. A combinatorial coincidence problem, 955.

Metcalf, F. T. See Diaz, J. B.

Meyer, K. R. Periodic points of diffeomorphisms, 615.

Meyer-Konig, Werner and Tietz, Hubert. On Tauberian conditions of type o, 926.

Milgram, R. James. See Mahowald, M.

Minty, George J. On the generalization of a direct method of the calculus of variations, 315.

Mitchell, Josephine. See Hahn, K. T.

Mond, B. See Shisha, O.

Monk, J. Donald. Nontrivial m-injective Boolean algebras do not exist, 526.

Moody, R. V. Lie algebras associated with generalized Cartan matrices, 217.

Moore, R. T. Duality methods and perturbation of semigroups, 548.

Nadkarni, M. See Mandrekar, V.

Namioka, I. and Asplund, E. A geometric proof of Ryll-Nardzerwski's fixed point theorem, 443.

Narasimhan, Raghavan. See Chandrasekharan, K.

Nehari, Zeev, Extremal problems for a class of functionals defined on convex sets, 584 .

Neuberger, J. W. Symmetry in non self adjoint Sturm-Liouville systems, 701.

Nijenhuis, Albert, $A$ Lie product for the cohomology of subalgebra with coefficients in the quotient, 962.

- and Richardson, R. W. Jr. Deformations of homomorphisms of Lie groups and Lie algebras, 175.

Nirenberg, Ricardo and Wells, R. O. Jr. Holomorphic approximation on real submanifolds of a complex manifold, 378.

O'Neill, Duane, Duality and orientability in bordism theories, 545. 
Opial, Zdzislaw. Weak convergence of the sequence of successive approximations for nonexpansive mappings, 591.

Osher, Stanley J. Canonical forms of certain Volterra integral operators and a method of solving the commutator equations which involve them, 423.

Palmer, Edgar M. and Robinson, R. W., The matrix group of two permutation groups 204.

Parry, W. Principal partitions and generators, 307.

Pedersen, E. A. See Porcelli, P.

Petrich, Mario. See Lallement, Gerard.

Petrie, Ted. The Adams spectral sequence for $U^{*}\left(X, Z_{p}\right)$ and applications to Lie groups, etc. 671.

The weakly complex bordism of Lie groups, 689 .

Phillips, R. E. On direct products of generalized solvable groups, 973 .

Pinchuk, Bernard. Extremal problems for functions of bounded boundary rotation, 708 .

Pincus, Joel David. Singular integral operators on the unit circle, 195.

Pitcher, Everett. See Khabbaz, Samir.

Porcelli, P. and Pedersen, E. A. On rings of operators, 142.

Pour-El, Marian Boykan, and Kripke, Saul. Deduction-preserving "recursive isomorphisms" between theories, 145.

Powers, Robert T. Representations of uniformly hyperfinite algebras and their associated von Neumann rings, 572.

Putnam, C. R. Wiener-Hopf operators and absolutely continuous spectra, 659.

Rajagopalan, M. and Soundararajan, T. On self dual L C A groups, 985.

Ralley, T. G. See Hannula, T. A. and Reiner, I.

Rauch, H. E. The local ring of the genus three modulus space at Klein's 168 surface, 343.

The vanishing of a theta constant is peculiar phenomenon, 339 .

Reiner, I. See Hannula, T. A. and Ralley, T. G.

Retherford, J. R. On Čebyšev subspaces and unconditional bases in Banach spaces, 238.

Richardson, R. W. Jr. See Nijenhuis, Albert.

Richter, Wayne. Constructive transfinite number classes, 261.

Rickert, Neil W. The range of a measure, 560 .

Rivlin, T. J. Some explicit polynomial approximations in the complex domain, 467.

Robinson, R. W. See Palmer, Edgar M.

Rosenthal, P. See Crimmins, T.

Rothschild, Bruce. Degrees of irreducible modular characters of blocks with cyclic defect groups, 102.

Rudin, Walter. Inner functions in polydiscs, 369.

Zero-sets in polydiscs, 580.

Sacks, Gerald E. Measure-theoretic uniformity, 169.

Schechter, Martin. Essential spectra of elliptic partial differential equations, 567.

- Intermediate extensions in $L^{p}, 979$.

Schmets, J. See Garnir, H. G.

Schurle, Arlo W. Compactification of strongly countable dimensional spaces, 909.

Schweizer, B. and Sklar, A. The algebra of multiplace vector-valued functions, 510.

Seebeck, C. L. Jr. See Harrold, O. G. Jr.

Seligman, George B. Some results on Lie p-algebras, 528.

Shapiro, H. S. See Douglas, R. G. and Shields, A. L.

Shields, A. L., See Douglas, R. G., and Shapiro, H. S.

Shisha, O. and Mond, B. Differences of means, 328.

Simmons, James. A note on minimal varieties, 491. 
Singer, William M. Connective fiberings over BU and U, 897.

Singletary, W. E. The equivalence of some general combinatorial decision problems, 446.

Sklar, A. See Schweizer, B.

Small, Lance W. Semihereditary rings, 656.

Smith, Larry, Multiplicative fibre maps, 334.

Smoller, J. A. See Johnson, J. L.

Snell, J. L. See Kemeny, J. G.

Soundararajan, T. See Rajagopalan, M.

Speakman, Jane M.O. An algebraic characterisation of convergence ideals, 53.

Sprecher, David A. On the closure of certain Banach spaces of functions of several variables, 387.

Stakgold, I. Bounds for linear functionals, 180.

Stenger, William. An inequality for the eigenvalues of a class of self-adjoint operators, 487.

Stancl, D. L. Multiplication in Grothendieck rings of integral group rings, 92.

Stephens, N. M. Conjectures concerning elliptic curves, 160.

St $\phi$ mer, Erling. Two-sided ideals in $C^{*}$-algebras, 254.

Sucheston, Louis. On the ergodic theorem for positive operators, 403.

Sullivan, D. On the Hauptvermutung for manifolds, 598.

Sweedler, Moss E. Cocommutative Hopf algebras with antipode, 126.

Szeptycki, P. Some remarks on the extended domain of Fourier transform, 398.

Taft, Earl J. Cohomology of algebraic groups and invariant splitting of algebras, 106.

Taibleson, Mitchell H. Fourier series on the ring of integers in a p-series field, 623.

Talbot, Thomas D. See Loscalzo, Frank R.

Tamura, T. See Chrislock, J. L.

Taylor, B. A. See Kelleher, T.

Thompson, Richard B. On the semicomplexes of F. Browder, 531.

Tietz, Hubert. See Meyer-Konig, Werner.

Trudinger, Neil S. The Dirichlet problem for nonuniformly elliptic equations, 410.

Uhl, J. J. Orlicz spaces of finitely additive set functions, linear operators, and Martingales, 116.

Ullman, J. L. A problem of Schmidt and Spitzer, 883.

Wagoner, J. B. Producing PL homeomorphisms by surgery, 78. Smooth and piecewise linear surgery, 72.

Warren, Hugh E. Partitions by real algebraic varieties, and applications to questions of nonlinear approximation, 192.

Warne, R. J. Erratum to On certain bisimple inverse semigroups, 496.

Warner, Seth. Compactly generated algebras over discrete fields, 227. Locally compact semilocal rings, 906.

Waterman, Daniel. On the summability of the differentiated Fourier series, 109.

Weidmann, Joachim. The virial theorem and its application to the spectral theory Schrödinger operators, 452.

Wells, R. O. Jr. See Nirenberg, Ricardo.

Wilcox, Theodore W. On the structure of maximally almost periodic groups, 732.

Wilhelmsen, R. A nonlinear boundary value problem, 920.

Williams, David. Local time at fictitious states, 542. On local time for Markov chains, 432.

Wolkowisky, Jay H. A nonlinear Sturm-Liouville problem, 634.

Young, S. W. Piecewise monotone polynomial interpolation, 642.

Zachariou, A. $A$ subalgebra of Ext $A^{* *}\left(Z_{2}, Z_{2}\right), 647$. 
Zeeman, E. C. See Armstrong, M.A.

Zemanian, A. H. The Weierstrass transformation of certain generalized functions, 682. Zieschang, H. See Burde G.

\section{BOOK REVIEWS}

Feldman, Jacob. Review of "Mathematical foundations of quantum mechanics" by $G$. W. Mackey, 499.

Herstein, I. N. Review of "Lectures in abstract algebra, Vol. III. Theory of fields and Galois theory" by Nathan Jacobson, 44.

Nachbin, Leopoldo. Review of "Abstract harmonic analysis" by Edwin Hewitt and K. A. Ross, 292.

\section{REPORTS OF MEETINGS AND MISCELLANEOUS}

Bateman, Paul T. Reports of Meetings of the American Mathematical Society: The April Meeting in Chicago, 508.

See Pitcher, Everett.

By-Laws of the American Mathematical Society, 988.

Brauer, Richard. Emil Artin, 27.

Council and Board of Trustees, 1966, 189.

Federer, Herbert. Reports of Meetings of the American Mathematical Society: The February Meeting in New York, 306. See Pitcher, Everett.

Harrold, O. G. Reports of Meetings of the American Mathematical Society: The November Meeting in Tampa, 48.

Pierce, R. S. Reports of Meetings of the American Mathematical Society: The April Meeting in San Jose, 509; The June Meeting in Missoula, 614. The November Meeting in La Jolla, 49.

Pitcher, Everett. Reports of Meetings of the American Mathematical Society: The October Meeting in Philadelphia, 47; The November Meeting in Mexico City, 191.

Pitcher, Everett and Bateman, Paul T. Reports of Meetings of the American Mathematical Society: The Annual Meeting in Houston, 295.

Pitcher, Everett and Federer, Herbert. Reports of Meetings of the American Mathematical Society: The April Meeting in New York, 501; The Summer Meeting in Toronto, 844 .

Report of the Treasurer, by W. T. Martin, 50.

Smale, S. Differentiable dynamical systems, 747.

Statement of Ownership. Management \& Circulation, 987.

\section{INVITED ADDRESSES}

Accola, Robert D. M. Some classical theorems on open Riemann surfaces, 13.

Fuchs, W. H. J. Developments in the classical Nevanlinna theory of meromorphic functions, 275.

Glauberman, George. Subgroups of finite groups, 1.

Neumann, B. H. Varieties of groups, 603.

Robinson, Abraham. Nonstandard arithmetic, 818. 